\title{
LIE SYMMETRIES OF FIRST ORDER NEUTRAL DIFFERENTIAL EQUATIONS
}

\author{
Jervin Zen Lobo ${ }^{1}$, Y.S. Valaulikar ${ }^{2}$ \\ ${ }^{1}$ Department of Mathematics, St. Xavier's College \\ Mapusa, Goa - 403507, India \\ ${ }^{2}$ Department of Mathematics, Goa University \\ Taleigao Plateau, Goa - 403206, India \\ zenlobo1990@gmail.com,ysv@unigoa.ac.in
}

Received: 10 October 2018; Accepted: 16 April 2019

\begin{abstract}
In this paper we extend the method of obtaining symmetries of ordinary differential equations to first order non-homogeneous neutral differential equations with variable coefficients. The existing method for delay differential equations uses a Lie-Bäcklund operator and an Invariant Manifold Theorem to define the operators which are used to obtain the infinitesimal generators of the Lie group. In this paper, we adopt a different approach and use Taylor's theorem to obtain a Lie type invariance condition and the determining equations for a neutral differential equation. We then split this equation in a manner similar to that of ordinary differential equations to obtain an over-determined system of partial differential equations. These equations are then solved to obtain corresponding infinitesimals, and hence desired equivalent symmetries. We then obtain the symmetry algebra admitted by this neutral differential equation.
\end{abstract}

MSC 2010: 22E65, 22E70, 76M60, 58J70

Keywords: determining equations, infinitesimals, invariance, neutral differential equations, splitting equations, symmetries

\section{Introduction}

In many branches of science and engineering, differential equations are of profound importance as we encounter them in modelling most physical phenomenon. However, differential equations involve the values of the unknown function and its derivatives, all at the same instant $t$. This may not always be the case, as most systems respond after a time delay. When the derivative of a function, at a given time instant, depends not only on the unknown function values at the same instant, but also at previous instants. Such equations are then called Delay Differential Equations. Delay differential equations find applications in biological systems, population dynamics, networking problems, rolling of ships, electrical engineering, etc [1]. Well known methods to solve delay differential equations are method of steps, numerical 
solutions, substitutions, and power series solutions [2]. A lot of literature on delay differential equations is available in $[3,4]$.

A more general class of differential equations are ones with deviating arguments, i.e equations containing some functions along with some of their derivatives at different argument values. This class of differential equations are called Functional Differential Equations. The various types of functional differential equations are differential difference equations, integro-differential equations, delay differential equations, neutral differential equations, etc. Several applications of functional differential equations include heat transfer problems, signal processing, evolution of species, traffic flow, study of epidemics, population models, prey-predator models, etc.

In this paper we restrict our attention to neutral differential equations. Neutral differential equations are differential equations in which the unknown function and its derivative appear with time delays. Such equations are of importance in models involving flip-flop circuits [5], compartmental systems [6], etc. and are extensively studied. In [7], neutral differential equations are solved using the multistep block method. Other methods of solution include the implicit block method [8], and analyse discontinuities of the derivatives as studied in [9]. Our focus is to obtain symmetries and the corresponding generators of neutral differential equations.

Symmetries are transformations that leave an object unchanged or invariant. As explained in [10], symmetries are very useful in the formation and study of laws of nature. They find a wide range of applications in Physics and Mathematics in the existence of conservation laws. The need for similarity arises from the regularities of the laws that are independent of some inessential circumstances. The reproducibility of experiments in different places and different times rely on invariance laws. The concept of symmetry has interested many scientists, like Kepler, in determining the orbits of planets, to Newton in studying the laws of mechanics - as a symmetry principle. In the nineteenth century, Sophus Lie began investigations of continuous groups of transformations leaving the differential equation invariant, in a study now called symmetry analysis of differential equations. The main idea in Lie's theory of symmetry analysis of differential equations relies on the invariance of the latter under a transformation of dependent and independent variables.

In this paper, we study the first order neutral differential equation

$$
x^{\prime}(t)=f\left(t, x(t), x\left(t_{1}\right), x^{\prime}\left(t_{1}\right)\right),
$$

where $f$ is defined on a suitable domain discussed in detail in the subsequent sections. The notations $x\left(t_{1}\right)$ mean $x(g(t)), g(t)<t$ and $x^{\prime}\left(t_{1}\right)$ mean $\frac{d x}{d t}(g(t))$. We further assume that $\frac{\partial f}{\partial x\left(t_{1}\right)} \neq 0$ and $\frac{\partial f}{\partial x^{\prime}\left(t_{1}\right)} \neq 0$. To determine the problem completely, we specify the delay point $t_{1}$ by $t_{1}=g(t)$, where $g(t)<t$, which is the most general kind of delay. We assume that the delay function $g(t)$ is sufficiently smooth in some interval. We will first need to find a group under which this neutral differential equation is invariant. We call this the admitted Lie group by which we mean that one solution 
curve is carried to another solution curve of the same equation. We then use this group to obtain the desired equivalent symmetries.

Several research papers $[11,12]$, are dedicated to obtaining symmetries of delay differential equations. These research papers define a certain operator, equivalent to the canonical Lie-Bäcklund operator, which they use for obtaining symmetries. A research paper by Pue-on [13] is devoted to obtain equivalent symmetries of a second order delay differential equation by defining an operator equivalent to the canonical Lie-Bäcklund operator and suitable other operators. Linchuk [14] has suggested a group method to study functional differential equations based on a search of symmetries of underdetermined differential equations by methods of classical and modern group analysis, using the principle of factorization. The method therein encompasses the use of a basis of invariants consisting of universal and differential invariants. A classification of some non-linear neutral differential equations with constant coefficients to solvable Lie algebras are made in [15]. Recently in [16] Lie symmetry method is employed to solve and obtain the infinite dimensional symmetry algebras of fractional neutral ordinary differential equations. In this paper, we extend the results of obtaining symmetries of ordinary differential equations $[17,18]$ to obtain symmetries of first order non-homogeneous neutral differential equations with variable coefficients and general delay. We use Taylor's theorem to do this. We then obtain a Lie type invariance condition for neutral differential equations. Using this, we suitably define an operator, its prolongation and extension and use it to obtain our determining equations. These equations are then split with respect to the independent variables to obtain an over-determined system of partial differential equations, which are then solved to obtain the most general generator of the Lie group and the corresponding symmetries. We can then show that this neutral differential equation admits an infinite dimensional Lie algebra.

\section{Preliminaries}

We first present some definitions and construction of infinitesimals for ordinary differential equations.

\section{Definition 1 (Lie Group)}

Consider transformations $\bar{t}_{i}=f_{i}\left(t_{j}, \delta\right), i, j=1,2, \cdots, n$, which continuously depend on the parameter $\delta$. Further, we assume that for each $i, f_{i}$ is a smooth function of the variables $t_{j}$ and has convergent Taylor series in $\delta$.

This set of transformations are said to form a group if:

(i) Two transformations carried out in succession are equivalent to another transformation of the set.

(ii) There is a transformation for which the source and image points coincide.

(iii) Each transformation has an inverse. 
In general, the order in which the transformations are carried out matters. If the order does not matter, then we label the group as abelian.

For example, $\bar{t}=a^{\delta} t, t \in \mathbb{R} \backslash\{0\}$ is a one parameter group called the stretching group. As another example, consider $\bar{t}=t+\delta$, which is also a group called the translational group.

For a first order ordinary differential equation $\frac{d x}{d t}=F(t, x)$, if $\bar{t}=f_{1}(t, x ; \delta)$, $\bar{x}=f_{2}(t, x ; \delta)$, then $\omega(t, x)=\frac{\partial f_{1}(t, x ; 0)}{\partial \delta}$ and $\Upsilon(t, x)=\frac{\partial f_{2}(t, x ; 0)}{\partial \delta}$.

$\omega$ and $\Upsilon$ are called coefficients of the infinitesimal transformations or simply infinitesimals. The infinitesimal generator is given by, $\zeta^{*}=\omega(t, x) \frac{\partial}{\partial t}+\Upsilon(t, x) \frac{\partial}{\partial x}$. Let the differential equation be invariant under the Lie group

$$
\bar{t}=t+\delta \omega(t, x)+O\left(\delta^{2}\right), \quad \bar{x}=x+\delta \Upsilon(t, x)+O\left(\delta^{2}\right) .
$$

If we express our differential equation as $\frac{d \bar{x}}{d \bar{t}}=F(\bar{t}, \bar{x})$.

Then,

$$
\begin{aligned}
\frac{d \bar{x}}{d \bar{t}} & =\frac{\frac{d \bar{x}}{d t}}{\frac{d \bar{t}}{d t}} \\
& =\frac{\frac{d}{d t}\left(x+\delta \Upsilon(t, x)+O\left(\delta^{2}\right)\right.}{\frac{d}{d t}\left(t+\delta \omega(t, x)+O\left(\delta^{2}\right)\right.} \\
& =\frac{\frac{d x}{d t}+\left(\Upsilon_{t}+\Upsilon_{x} x^{\prime}\right) \delta+O\left(\delta^{2}\right)}{1+\left(\omega_{t}+\omega_{x} x^{\prime}\right) \delta+O\left(\delta^{2}\right)} \\
& =\left[\frac{d x}{d t}+\left(\Upsilon_{t}+\Upsilon_{x} x^{\prime}\right) \delta+O\left(\delta^{2}\right)\right]\left[1-\left(\omega_{t}+\omega_{x} x^{\prime}\right) \delta+O\left(\delta^{2}\right)\right] \\
& =\frac{d x}{d t}+\left[\Upsilon_{t}+\left(\Upsilon_{x}-\omega_{t}\right) x^{\prime}-\omega_{x} x^{\prime 2}\right] \delta+O\left(\delta^{2}\right) .
\end{aligned}
$$

Using the fact that the differential equation is invariant under the above Lie group, we get

$$
\Upsilon_{t}+\left(\Upsilon_{x}-\omega_{t}\right) F-\omega_{x} F^{2}=\omega F_{t}+\Upsilon F_{x},
$$

which is known as Lie's Invariance condition.

The above preliminaries for ordinary differential equations stands a motivation for our work on neutral differential equations as corresponding literature for delay differential equations is still in developing stages on different lines.

We have the following definitions:

Definition 2 (Neutral Differential Equation)

Let $J$ be an interval in $\mathbb{R}$, and let $D$ be an open set in $\mathbb{R}$. Sometimes $J$ will be $\left[t_{0}, \beta\right)$, and sometimes it will be $(\alpha, \beta)$, where $\alpha \leq t_{0} \leq \beta$. Let $f \operatorname{map} J \times D^{3} \rightarrow \mathbb{R}$. 
Conveniently, a first order neutral differential equation is expressed as

$$
x^{\prime}(t)=f\left(t, x(t), x(g(t)), x^{\prime}(g(t))\right),
$$

where $x$ and $f$ are real valued functions, and $g(t)$ is a retarded argument i.e. $g(t) \leq t$. We consider equation (4) for $t_{0} \leq t \leq \beta$ together with the initial function

$$
x(t)=\theta(t), \gamma \leq t \leq t_{0},
$$

where $\theta$ is a given initial function mapping $\left[\gamma, t_{0}\right] \rightarrow D$.

\section{Definition 3 (Solution of a Neutral Differential Equation)}

By a solution of the neutral differential equation (4) satisfying (5), we mean a differentiable function $x:\left[\gamma, \beta_{1}\right) \rightarrow D$, for some $\beta_{1} \in\left(t_{0}, \beta\right]$, such that

1. $x(t)=\theta(t)$, for $\gamma \leq t \leq t_{0}$, and

2. $x(t)$ reduces equation (4) to an identity on $t_{0} \leq t \leq \beta_{1}$.

We understand $x^{\prime}\left(t_{0}\right)$ to mean the right-hand derivative.

\section{Extension of results and Lie type invariance condition for Neutral Differential Equations}

In this section we extend the results of ordinary differential equations to neutral differential equations given by equation (1). In order to determine the neutral differential equation completely, we need to specify the delay term, where the delayed function is specified, otherwise the problem is not fully determined.

Let a function $\mathrm{F}$ be defined on a 5-dimensional space. We extend our results to

$$
\frac{d x}{d t}=F\left(t, x, g(t), x(g(t)), x^{\prime}(g(t))\right) .
$$

Let the neutral differential equation be invariant under the Lie group

$$
\bar{t}=t+\delta \omega(t, x)+O\left(\delta^{2}\right), \quad \bar{x}=x+\delta \Upsilon(t, x)+O\left(\delta^{2}\right) .
$$

We then naturally define $\overline{g(t)}=g(t)+\delta \omega(g(t), x(g(t)))+O\left(\delta^{2}\right)$ and $\overline{x(g(t))}=$ $=x(g(t))+\delta \Upsilon(g(t), x(g(t)))+O\left(\delta^{2}\right)$.

With the notations, $\omega_{1}=\omega(g(t), x(g(t)))$, and $\left.\Upsilon_{1}=\Upsilon(g(t), x(g(t)))\right)$, it follows that,

$$
\begin{aligned}
\bar{x}^{\prime}\left(t_{1}\right) & =\frac{d \bar{x}}{d \bar{t}}(\overline{g(t)}) \\
& =x^{\prime}\left(t_{1}\right)+\left(\left(\Upsilon_{1}\right)_{t_{1}}+\left(\left(\Upsilon_{1}\right)_{x\left(t_{1}\right)}-\left(\omega_{1}\right)_{t_{1}}\right) x^{\prime}\left(t_{1}\right)-\left(x^{\prime}\left(t_{1}\right)\right)^{2}\left(\omega_{1}\right)_{x\left(t_{1}\right)}\right) \delta+O\left(\delta^{2}\right) .
\end{aligned}
$$

For invariance, $\frac{d \bar{x}}{d \bar{t}}=F\left(\bar{t}, \bar{x}, \overline{g(t)}, \overline{(x(g(t)))}, \overline{x^{\prime}(g(t))}\right)$. 
This gives,

$$
\begin{aligned}
& \frac{d x}{d t}+\left[\Upsilon_{t}+\left(\Upsilon_{x}-\omega_{t}\right) x^{\prime}-\omega_{x} x^{\prime 2}\right] \delta+O\left(\delta^{2}\right) \\
= & F\left(t+\delta \omega+O\left(\delta^{2}\right), x+\delta \Upsilon+O\left(\delta^{2}\right), g(t)+\delta \omega_{1}+O\left(\delta^{2}\right), x\left(t_{1}\right)+\delta \Upsilon_{1}+O\left(\delta^{2}\right),\right. \\
& \left.x^{\prime}\left(t_{1}\right)+\left(\left(\Upsilon_{1}\right)_{t_{1}}+\left(\left(\Upsilon_{1}\right)_{x\left(t_{1}\right)}-\left(\omega_{1}\right)_{t_{1}}\right) x^{\prime}\left(t_{1}\right)-\left(x^{\prime}\left(t_{1}\right)\right)^{2}\left(\omega_{1}\right)_{x\left(t_{1}\right)}\right) \delta+O\left(\delta^{2}\right)\right) \\
= & F\left(t, x, g(t), x(g(t)), x^{\prime}(g(t))\right)+\left(\omega F_{t}+\Upsilon F_{x}+\omega_{1} F_{t_{1}}+\Upsilon_{1} F_{x\left(t_{1}\right)}\right. \\
& \left.+\Upsilon_{1[t]} F_{x^{\prime}\left(t_{1}\right)}\right) \delta+O\left(\delta^{2}\right),
\end{aligned}
$$

where $\Upsilon_{1[t]}=\left(\Upsilon_{1}\right)_{t_{1}}+\left(\left(\Upsilon_{1}\right)_{x\left(t_{1}\right)}-\left(\omega_{1}\right)_{t_{1}}\right) x^{\prime}\left(t_{1}\right)-\left(x^{\prime}\left(t_{1}\right)\right)^{2}\left(\omega_{1}\right)_{x\left(t_{1}\right)}$

Comparing the coefficient of $\delta$, we get

$$
\omega F_{t}+\Upsilon F_{x}+\omega_{1} F_{t_{1}}+\Upsilon_{1} F_{x\left(t_{1}\right)}+\Upsilon_{1[t]} F_{x^{\prime}\left(t_{1}\right)}=\Upsilon_{t}+\left(\Upsilon_{x}-\omega_{t}\right) x^{\prime}-\omega_{x} x^{\prime 2}
$$

The above obtained equation (9) is a Lie type invariance condition.

Similar to the case of ordinary differential equations, we can define a prolonged operator for neutral differential equation as

$$
\zeta=\omega \frac{\partial}{\partial t}+\omega_{1} \frac{\partial}{\partial t_{1}}+\Upsilon \frac{\partial}{\partial x}+\Upsilon_{1} \frac{\partial}{\partial x\left(t_{1}\right)} .
$$

With the notation $D_{t}=\frac{\partial}{\partial t}+x^{\prime} \frac{\partial}{\partial x}$, we can write,

$$
\begin{aligned}
\frac{d \bar{x}}{d \bar{t}} & =\frac{d x}{d t}+\left(D_{t}(\Upsilon)-x^{\prime} D_{t}(\omega)\right) \delta+O\left(\delta^{2}\right) \\
& =\frac{d x}{d t}+\Upsilon_{[t]} \delta+O\left(\delta^{2}\right)
\end{aligned}
$$

where $\Upsilon_{[t]}=D_{t}(\Upsilon)-x^{\prime} D_{t}(\omega)$. We then define the extended operator

$$
\zeta^{(1)}=\omega \frac{\partial}{\partial t}+\omega_{1} \frac{\partial}{\partial t_{1}}+\Upsilon \frac{\partial}{\partial x}+\Upsilon_{1} \frac{\partial}{\partial x\left(t_{1}\right)}+\Upsilon_{[t]} \frac{\partial}{\partial x^{\prime}}+\Upsilon_{1[t]} \frac{\partial}{\partial x^{\prime}\left(t_{1}\right)}
$$

Defining $\Delta=x^{\prime}(t)-F\left(t, x(t), g(t), x(g(t)), x^{\prime}(g(t))\right)=0$, we get

$$
\zeta^{(1)} \Delta=\Upsilon_{[t]}-\omega F_{t}+\Upsilon F_{x}+\omega_{1} F_{t_{1}}+\Upsilon_{1} F_{x\left(t_{1}\right)}+\Upsilon_{1[t]} F_{x^{\prime}\left(t_{1}\right)} .
$$

Comparing equations (9) and (12), we get

$\Upsilon_{[t]}=\Upsilon_{t}+\left(\Upsilon_{x}-\omega_{t}\right) x^{\prime}-\omega_{x} x^{\prime 2}$.

On substituting $x^{\prime}=F$ into $\zeta^{(1)} \Delta=0$, we get an invariance condition for the neutral differential equation which is $\left.\zeta^{(1)} \Delta\right|_{\Delta=0}=0$, from which we will obtain the determining equations.

We point out here that equations (10)-(12) is an easy way of working we higher order differential equations as compared to equations (7)-(9) which is simpler to use for lower order differential equations. 


\section{Symmetries of Non-homogeneous Neutral Differential Equation of First Order}

Consider the neutral differential equation

$$
x^{\prime}(t)=\alpha(t) x(t)+\beta(t) x(g(t))+\gamma(t)+\rho(t) x^{\prime}(g(t)) .
$$

We obtain symmetries of the non-homogeneous neutral differential equation (13), where $g$ is a sufficiently smooth function with $g(t)<t$. Also $\alpha(t), \beta(t), \gamma(t)$ and $\rho(t)$ are sufficiently smooth functions satisfying $\beta^{2}(t)+\rho^{2}(t)$ not identically zero and $g(t)$ is non constant.

We seek our coefficient of the infinitesimal transformation $\omega$ of the form, (that is we assume) $\omega(t, x)=\omega(t)$. Then applying the operator defined by (11), to the delay equation $t_{1}=g(t)$, we get,

$$
\omega_{1}=g^{\prime}(t) \omega(t)
$$

Applying operator $\zeta^{(1)}$ defined by (11) to equation (13), we get

$$
\begin{aligned}
& \Upsilon_{t}(t, x)+\left(\Upsilon_{x}(t, x)-\omega^{\prime}(t)\right)\left(\alpha(t) x(t)+\beta(t) x(g(t))+\gamma(t)+\rho(t) x^{\prime}(g(t))=\right. \\
& \omega(t)\left(\alpha^{\prime}(t) x(t)+\beta^{\prime}(t) x(g(t))+\gamma^{\prime}(t)+\rho^{\prime}(t) x^{\prime}(g(t))\right)+\alpha(t) \Upsilon(t, x)+\beta(t) \Upsilon_{1} \\
& +\rho(t)\left(\left(\Upsilon_{1}\right)_{t_{1}}+\left(\left(\Upsilon_{1}\right)_{x\left(t_{1}\right)}-\omega_{1}^{\prime}\right)\right)\left(\alpha(t) x(t)+\beta(t) x(g(t))+\gamma(t)+\rho(t) x^{\prime}(g(t))\right) .
\end{aligned}
$$

Differentiating with respect to $x\left(t_{1}\right)$ twice, we get

$\beta(t)\left(\Upsilon_{1}\right)_{x\left(t_{1}\right) x\left(t_{1}\right)}+\rho(t)\left[\left(\Upsilon_{1}\right)_{t_{1} x\left(t_{1}\right) x\left(t_{1}\right)}+(\alpha(t) x(t)+\beta(t) x(g(t))+\gamma(t)\right.$

$\left.\left.+\rho(t) x^{\prime}(g(t))\right)\left(\Upsilon_{1}\right)_{x\left(t_{1}\right) x\left(t_{1}\right) x\left(t_{1}\right)}\right]=0$.

Splitting the equation with respect to $x^{\prime}(g(t))$, we get $\rho^{2}(t)\left(\Upsilon_{1}\right)_{x\left(t_{1}\right) x\left(t_{1}\right) x\left(t_{1}\right)}=0$, which is solved to give

$$
\Upsilon(t, x)=\frac{1}{2} A(t) x^{2}+B(t) x+C(t) .
$$

Substituting equation (16) into the determining equation (15), we get

$$
\begin{aligned}
& \frac{1}{2} A^{\prime}(t) x^{2}+B^{\prime}(t) x+C^{\prime}(t)+\left(A(t) x+B(t)-\omega^{\prime}(t)\right)(\alpha(t) x \\
& \left.+\beta(t) x(g(t))+\gamma(t)+\rho(t) x^{\prime}(g(t))\right) \\
& =\omega(t)\left(\alpha^{\prime}(t) x+\beta^{\prime}(t) x(g(t))+\gamma^{\prime}(t)+\rho^{\prime}(t) x^{\prime}(g(t))\right)+\alpha(t)\left(\frac{1}{2} A(t) x^{2}\right. \\
& +B(t) x+C(t))+\beta(t)\left(\frac{1}{2} A\left(t_{1}\right) x^{2}\left(t_{1}\right)+B\left(t_{1}\right) x\left(t_{1}\right)+C\left(t_{1}\right)\right) \\
& +\rho(t)\left[\left(\frac{1}{2} A^{\prime}\left(t_{1}\right) x^{2}\left(t_{1}\right)+B^{\prime}\left(t_{1}\right) x\left(t_{1}\right)+C^{\prime}\left(t_{1}\right)\right)+\left(A\left(t_{1}\right) x\left(t_{1}\right)+B\left(t_{1}\right)\right.\right. \\
& \left.\left.-\omega_{1}^{\prime}\left(t_{1}\right)\right)\left(\alpha(t) x+\beta(t) x(g(t))+\gamma(t)+\rho(t) x^{\prime}(g(t))\right)\right] .
\end{aligned}
$$

Splitting equation (17) with respect to $x^{2}$, we get

$$
A(t)=\exp \left(-\int^{t} \alpha(s) d s\right)+A_{0}
$$


where $A_{0}=$ constant.

Similarly splitting equation (17) with respect to $x, x(g(t)), x^{2}(g(t)), x^{\prime}(g(t))$, $x(t) x(g(t)), x(t) x^{\prime}(g(t)), x(g(t)) x^{\prime}(g(t))$ and with respect to constant term, we get

$$
\begin{gathered}
B^{\prime}(t)+A(t) \gamma(t)-\rho(t) \alpha(t) B\left(t_{1}\right)=\omega^{\prime}(t) \alpha(t)+\alpha^{\prime}(t) \omega(t)-\rho(t)\left(\omega_{1}\right)^{\prime}\left(t_{1}\right) \alpha(t) \\
\rho(t) B^{\prime}\left(t_{1}\right)+\left(B\left(t_{1}\right)(1+\rho(t))-B(t)\right) \beta(t)+\rho(t) A\left(t_{1}\right) \gamma(t) \\
-\rho(t)\left(\omega_{1}\right)^{\prime}\left(t_{1}\right) \beta(t)+\omega^{\prime}(t) \beta(t)+\omega(t) \beta^{\prime}(t)=0 \\
A\left(t_{1}\right) \beta(t)+\rho(t) A^{\prime}\left(t_{1}\right)+2 \rho(t) A\left(t_{1}\right) \beta(t)=0 \\
B(t) \rho(t)=\omega^{\prime}(t) \rho(t)+\omega(t) \rho^{\prime}(t)+\rho^{2}(t)\left(B\left(t_{1}\right)-\left(\omega_{1}\right)^{\prime}\left(t_{1}\right)\right) \\
A(t) \beta(t)=\rho(t) A\left(t_{1}\right) \alpha(t) \\
A(t) \rho(t)=0 \\
\rho^{2}(t) A\left(t_{1}\right)=0
\end{gathered}
$$

and

$$
\begin{aligned}
& C^{\prime}(t)+B(t) \gamma(t)-\omega^{\prime}(t) \gamma(t)=\omega(t) \gamma^{\prime}(t)+\alpha(t) C(t)+\beta(t) C\left(t_{1}\right) \\
& +\rho(t) C^{\prime}\left(t_{1}\right)+\rho(t) B\left(t_{1}\right) \gamma(t)-\rho(t)\left(\omega_{1}\right)^{\prime}\left(t_{1}\right) \gamma(t)
\end{aligned}
$$

respectively.

For a general $\alpha(t), \beta(t), \gamma(t), \rho(t)$ and $g(t)$, equations (14), (19), (20), (22) and (26) have only one solution namely, $\omega(t, x)=0$.

Equations (24) and (25), give $A(t)=0$.

With $\omega(t, x)=0$ and $A(t)=0$, equation (19) gives

$$
B^{\prime}(t)=\alpha(t) \rho(t) B\left(t_{1}\right) .
$$

Equation (20) gives

$$
\rho(t) B^{\prime}\left(t_{1}\right)+\left(B\left(t_{1}\right)(1+\rho(t))-B(t)\right) \beta(t)=0 .
$$

Equation (22) gives

$$
B(t)=\rho(t) B\left(t_{1}\right)
$$

Substituting equation (29) in equation (27), we get

$$
B(t)=B_{0} \exp \left(\int^{t} \alpha(s) d s\right),
$$


where $B_{0}=$ constant. Substituting equation (29) in equation (28), we get

$$
B(t) B^{\prime}\left(t_{1}\right)+B^{2}\left(t_{1}\right) \beta(t)=0 .
$$

Having obtained $B(t)$ from equation (30), equation (31) is the compatibility condition for $B(t)$, from which the value of $B_{0}$ can be found.

From equation (26), we get

$$
C^{\prime}(t)=\alpha(t) C(t)+\beta(t) C(g(t))+\rho(t) C^{\prime}(g(t)) .
$$

That is, $C(t)$ satisfies the corresponding homogeneous neutral differential equation. Thus, we obtain the coefficients of the symmetries as

$$
\omega(t, x)=0, \Upsilon(t, x)=B(t) x+C(t) .
$$

Hence the most general solution of the determining equations corresponds to the infinitesimal generator $\zeta^{*}=B(t) x \frac{\partial}{\partial x}+C(t) \frac{\partial}{\partial x}$, where $B(t)$ is given by equations (30), (31) and $C(t)$ solves the corresponding homogeneous neutral differential equation.

\section{Remarks:}

(1.) In obtainining equivalent symmetries of the neutral differential equation given by equation (13), we had assumed that, $\beta^{2}(t)+\rho^{2}(t)$ is not identically zero. However, we remark here that, if $\rho(t)=0, \beta(t) \neq 0$, then equation (13) reduces to a first order ordinary delay differential equation. From equation (26), we get $C^{\prime}(t)=\alpha(t) x(t)+\beta(t) x(g(t))-B(t) \gamma(t)$. From equation (27), we get, $B(t)=B_{0}$, a constant. Hence, the infinitesimal generator of the admitted Lie group in this case is given by, $\zeta^{*}=\left(B_{0} x+E(t)\right) \frac{\partial}{\partial x}$, where $E(t)$ is the solution of the delay differential equation $x^{\prime}(t)=\alpha(t) x(t)+\beta(t) x(g(t))-B_{0} \gamma(t)$.

(2.) If in equation (13), $\rho(t) \neq 0, \beta(t)=0$, then from equation (26), we get $C^{\prime}(t)=\alpha(t) x(t)-B(t) \gamma(t)(1-\rho(t))+\rho(t) C^{\prime}(g(t))$. From equation (31), $B(t)=B_{1}$, a constant. Hence the generator in this case is given by, $\zeta^{*}=\left(B_{1} x+G(t)\right) \frac{\partial}{\partial x}$, where $G(t)$ is the solution of equation $x^{\prime}(t)=\alpha(t) x(t)-B_{0} \gamma(t)(1-\rho(t))+\rho(t) x^{\prime}(g(t))$.

(3.) Further, if $\rho(t)=0, \beta(t)=0$, then equation (13), reduces to a first order ordinary differential equation. Again, From equation (27), we get, $B(t)=B_{2}$, a constant. Hence, the infinitesimal generator of the admitted Lie group in this case is given by, $\zeta^{*}=\left(B_{2} x+C_{0} \exp \left(\int^{t} \alpha(s) d s\right)\right) \frac{\partial}{\partial x}$. 


\section{Example}

We give an example to illustrate the construction of a Lie group under which a neutral differential equation is invariant.

Consider the neutral differential equation

$$
x^{\prime}(t)+x^{\prime}(g(t))=0 .
$$

Compared with (13), we get $\alpha(t)=\beta(t)=\gamma(t)=0$, and $\rho(t)=1$.

For a smooth $g(t)$ satisfying $g(t)<t, \quad x(t)=K$, a constant, is a solution of the equation (33). Following the procedure in section 4 , we see that, for a nonzero $B_{0}$,

$$
\omega(t, x)=0 \text { and } \Upsilon(t, x)=B_{0} x+K .
$$

This yields $\frac{d \bar{t}}{d \delta}=\omega(\bar{t}, \bar{x})=0$ and $\frac{d \bar{x}}{d \delta}=\Upsilon(\bar{t}, \bar{x})=B_{0} \bar{x}+K$.

On solving these equations with conditions $\bar{x}=x$ and $\bar{t}=t$, when $\delta=0$, we get

$$
\bar{t}=t \text { and } \bar{x}=\frac{1}{B_{0}}\left[\left(B_{0} x+K\right) e^{B_{0} \delta}-K\right]
$$

which is the Lie group under which neutral differential equation (33) is invariant.

\section{Conclusions}

We have obtained the symmetries of the first order non-homogeneous neutral differential equation with a general delay. We can make a group classification of the first order neutral differential equation into the following cases. In all cases we see that the first order neutral differential equation admits linear symmetries. The four cases are presented below:

(i) If $\rho(t) \neq 0, \beta(t) \neq 0$, and if $x_{1}(t)$ is a general solution of the associated homogeneous neutral differential equation, then the non-homogeneous neutral differential equation admits a symmetry algebra of infinite dimension, due to the linear superposition principle, given by vector fields $x_{1}(t) \frac{\partial}{\partial x}$ and $\left(x_{1}(t)-\exp \left(\int \alpha(s) d s\right) x\right) \frac{\partial}{\partial x}$.

(ii) If $\rho(t)=0, \beta(t) \neq 0$, and if $x_{2}(t)$ is a general solution of the associated homogeneous delay differential equation, then the non-homogeneous delay differential equation admits a symmetry algebra of infinite dimension, due to the linear superposition principle, given by vector fields $x_{2}(t) \frac{\partial}{\partial x}$ and $\left(x-x_{3}(t)\right) \frac{\partial}{\partial x}$, where $x_{3}(t)$ is the solution of the non-homogeneous delay differential equation $x^{\prime}(t)=\alpha(t) x(t)+\beta(t) x(g(t))+\gamma(t)$. Further, if the delay differential equation is homogeneous, then it admits a symmetry algebra again, of infinite dimension, given by vector fields $x_{2}(t) \frac{\partial}{\partial x}$ and $x \frac{\partial}{\partial x}$. 
(iii) If $\rho(t) \neq 0, \beta(t)=0$, and if $x_{4}(t)$ is a general solution of the associated homogeneous neutral differential equation, then the differential equation admits a symmetry algebra of infinite dimension, due to the linear superposition principle, given by vector fields, $x_{4}(t) \frac{\partial}{\partial x}$ and $\left(x-x_{5}(t)\right) \frac{\partial}{\partial x}$, where $x_{5}(t)$ is the solution of the non-homogeneous neutral differential equation $x^{\prime}(t)=\alpha(t) x(t)+\gamma(t)(1-\rho(t))-\rho(t) x^{\prime}(g(t))$.

(iv) If $\rho(t)=0, \beta(t)=0$, then the ordinary differential equation admits a symmetry algebra of infinite dimension, due to the linear superposition principle, given by vector fields, $x \frac{\partial}{\partial x}$ and $\left.\exp \left(\int^{t} \alpha(s) d s\right)\right) \frac{\partial}{\partial x}$.

\section{Acknowledgement}

Authors wish to thank the referees for their suggestions in improving the paper.

\section{References}

[1] Kyrychko, Y., \& Hogan, S.J. (2010). On use of delay equations in engineering applications. Journal of Vibrations and Control, 943-960.

[2] Deo, S.G., Lakshmikantham, V., \& Raghavendra, V. (2013). Text Book of Ordinary Differential Equations. McGraw Hill Education (India) Private Limited.

[3] Driver, R.D. (1977). Ordinary and Delay Differential Equations. New York: Springer-Verlag.

[4] Hale, J. (1977). Functional Differential Equations. New York: Springer-Verlag.

[5] Schmitt, K. (1972). Delay and Functional Differential Equations and their Applications. US Academic Press.

[6] Víctor, M.V., Novo, S., \& Obaya, R. (2008). Neutral functional differential equations with applications to compartmental systems. SIAM Journal on Mathematical Analysis, 40(3), 1003-1028.

[7] Seong, H.Y., \& Majid, Z.A. (2015). Solving neutral delay differential equations by using Multistep block method. IEEE Xplore.

[8] Ishak, F., \& Mohd, S. (2015). Implicit block method for solving neutral delay differential equations. AIP Conference Proceedings.

[9] Alfredo, B., \& Guglielmi, N. (2009). Solving neutral delay differential equations with state dependent delays. Journal of Computational and Applied Mathematics, 229, 350-362.

[10] Oliveri, F. (2010). Lie symmetries of differential equations: classical results and recent contributions. Symmetry Journal, 2(2), 658-706.

[11] Tanthanuch, J., \& Meleshko, S.V. (2002). Application of group analysis to delay differential equations. Proceedings of the 16th International Symposium on Nonlinear Acoustics. Moscow, Russia, 19-23 August, 607-610.

[12] Tanthanuch, J., \& Meleshko S.V. (2004). On definition of an admitted Lie group for functional differential equations. Communications in Nonlinear Science and Numerical Simulation, 9 , 117-125.

[13] Pue-on, P. (2009). Group classification of second order delay differential equations. Communications in Nonlinear Science and Numerical Simulation, 15, 1444-1453. 
[14] Linchuk, L.V. (2001). On group analysis of functional differential equations. Proceedings of the International Conference, MOGRAN 2000, 111-115.

[15] Muhsen, L., \& Maan, N. (2016). Lie group analysis of second-order non-linear neutral delay differential equations. Malaysian Journal of Mathematical Sciences, 10(S), 117-129.

[16] Nass, M.A. (2019). Lie symmetry analysis and exact solutions of fractional ordinary differential equations with neutral delay. Applied Mathematics and Computation, 347, 370-380.

[17] Arrigo, D. (2015). Symmetry Analysis of Differential Equations. New Jersy: John Wiley and Sons.

[18] Ibragimov, N.H. (1994, 1995, 1996), editor. CRC Handbook of Lie Group Analysis of differential equations, (Vol. 1, Vol. 2 and Vol. 3), Boca Raton: CRC Press. 